A N N A L E S Annales de Bretagne et des Pays de l'Ouest

Anjou. Maine. Poitou-Charente. Touraine

$115-4 \mid 2008$

Varia

\title{
De la terre à la mer, les frères Barbedienne
}

Jean-Michel Auffray

\section{OpenEdition}

Journals

Édition électronique

URL : http://journals.openedition.org/abpo/219

DOI : 10.4000/abpo.219

ISBN : 978-2-7535-1512-3

ISSN : 2108-6443

Éditeur

Presses universitaires de Rennes

Édition imprimée

Date de publication : 31 décembre 2008

Pagination : 81-109

ISBN : 978-2-7535-0808-8

ISSN : 0399-0826

Référence électronique

Jean-Michel Auffray, "De la terre à la mer, les frères Barbedienne », Annales de Bretagne et des Pays de I'Ouest [En ligne], 115-4 | 2008, mis en ligne le 31 décembre 2010, consulté le 20 avril 2019. URL http://journals.openedition.org/abpo/219; DOI : 10.4000/abpo.219 


\title{
De la terre à la mer, les frères Barbedienne
}

\author{
Jean-Michel AuFFRAY \\ Chercheur indépendant
}

\begin{abstract}
" À Pléneuf, les hommes sont grands, bien faits, processifs. Ils aiment à se donner de l'importance aussi les appelle-t-on les docteurs de Pléneuf... Les pâtoures ${ }^{1}$ y portent montre... Aucun ne veut servir en qualité de domestique après avoir passé l'âge de la conscription ${ }^{2}$."
\end{abstract}

Affirmations étonnantes qui peuvent prêter à sourire. Nous sommes en effet, au milieu du XIX ${ }^{\mathrm{e}}$ siècle, dans une commune rurale des Côtes-duNord, peu de temps après la famine des années 1847-1848 qui toucha si durement la Bretagne. La malnutrition, les épidémies sévissent encore et la tentation est grande de rejeter sans examen ces assertions. Si toutefois elles correspondent à une certaine réalité, on peut en déduire que, à Pléneuf, à cette époque, les hommes d'origine modeste n'avaient pas trop de difficultés pour assurer leur subsistance au point de refuser certains emplois de domestiques. Une des raisons de la relative prospérité de cette commune c'est la proximité du rivage. En effet, à une demi-heure à pied du bourg, il y a un port donnant sur la baie de Saint-Brieuc : Dahouët. À cette époque on pouvait y voir en avril quelques petits bâtiments pratiquant le cabotage, mais surtout des bricks et des trois mâts en partance pour Terre-Neuve.

L'importance de la grande pêche pour les communes littorales qui la pratiquaient n'est plus à démontrer. On évoque souvent Saint-Malo ou Paimpol qui ont basé leur développement sur cette activité au cours du $\mathrm{XIX}^{\mathrm{e}}$ siècle. Mais on oublie parfois qu'entre ces deux localités, la pêche à la morue a joué un rôle tout aussi fondamental pour les ports de la baie de Saint-Brieuc : Portrieux, Binic, Le Légué et Dahouët ${ }^{3}$. Là aussi, les tonnages étaient importants, les armements prospères et les marins

1. Ce sont probablement les pastoureaux.

2. OGÉE, Jean, Dictionnaire historique et géographique de la Bretagne. Édition de 1853.

3. QuerRé, Christian "Les Terre-Neuvas de la baie de Saint-Brieuc ", Le Chasse-marée, $\mathrm{n}^{\circ}$ 108, 1997, p 30-43; idem, La grande aventure des Terre-Neuvas de la baie de Saint-Brieuc, Binic, éditions du Dahin, 1998. 
recrutés nombreux. Sans doute, cependant, la baie de Saint-Brieuc a-telle moins bénéficié de l'impact médiatique et promotionnel des romans maritimes.

Quoi qu'il en soit, dans toutes ces régions côtières, le métier de marin offrait une perspective pour les fils de laboureurs parfois incapables de subvenir à leurs besoins : des revenus plus sûrs, plus importants. " La mer nourrit son homme " disait-on. Souvent plus sûrement que la terre, même si c'était au prix d'une existence très rude. Pendant plusieurs décennies, de jeunes paysans, contraints par les circonstances ou par besoin d'évasion, s'embarquent donc pour la grande pêche à Terre-Neuve ou à Islande. On les retrouve sur les registres de l'inscription maritime. Cependant, la grande variété des situations des inscrits soulève le problème de leur statut réel et de leur éventuelle pluriactivité. Dépendent-ils d'autres sources de revenus ou deviendront-ils des marins à part entière ${ }^{4}$ ? Dans ce dernier cas, comment le deviendront-ils? Le deviennent-ils définitivement? Quels en sont les déterminismes?

Des recherches généalogiques nous ont fait découvrir une famille de Pléneuf, les Barbedienne ${ }^{5}$, dont l'histoire pourrait illustrer ce questionnement et apporter quelques éléments de réponse. À l'instar de beaucoup d'autres, sa réussite sera limitée : ni promotions majeures ni retour à la misère, pas de catastrophe. En somme une famille ordinaire, une destinée banale peut-être, quoique nous ne puissions malheureusement rien savoir de la manière avec laquelle tous les acteurs de cette histoire ont vécu leurs réussites, leurs échecs, leurs joies, leurs drames. L'intérêt de cette famille tient au fait qu'elle est représentative d'une forme de pluriactivité rurale mais aussi de l'évolution de la pêche, de son importance et de ses mutations dans cette région à cette époque.

Ainsi, l'observation des parcours des frères Barbedienne ${ }^{6} \mathrm{a}$ été pour nous l'occasion de suivre l'évolution de l'armement à la grande pêche sur la côte nord de la Bretagne au XIX siècle : d'abord, reprise de la pêche sédentaire à Terre-Neuve puis, un peu après le milieu du siècle, du fait des difficultés rencontrées là-bas, reprise de la pêche errante sur les bancs de Terre-Neuve et à Islande. Durant toutes ces périodes, les armateurs transformeront en pêcheurs des générations de jeunes paysans sans qua-

4. Se pose, évidemment, l'épineuse question de la définition du marin qu'il n'est évidemment pas question de résoudre dans le cadre de cet article.

5. Pour identifier les membres de cette famille Barbedienne nous avons utilisé les registres de l'état-civil et les recensements de la commune de Pléneuf (XIXe siècle) déposés aux archives départementales des Côtes-d'Armor. Voir annexe : la famille Barbedienne à Pléneuf. Seuls y sont indiqués les enfants de Pierre Antoine Barbedienne et de Adélaïde Boschet qui ont atteint l'âge adulte.

6. Ce patronyme est très répandu à Pléneuf au début du siècle : certains Barbedienne d'autres branches seront maires ou capitaines. Pour éviter toute confusion, dans cet article les Barbedienne qui ne sont ni ascendants ni descendants directs de nos personnages centraux sont marqués d'un astérisque. Par la suite, la fréquence de ce nom diminue considérablement au fur et à mesure que d'autres patronymes apparaissent. 
lification. En marins aussi. Ces armateurs sont en effet moins spécialisés qu'une certaine tradition pourrait le laisser penser. Lorsque les problèmes de la pêche s'accroissent, on en voit certains réorienter une partie de leur activité vers le commerce international, à l'occasion, par exemple, d'un réarmement en Méditerranée : alors, certains équipages et certains navires armés au cabotage ou même au long cours, ne retournent plus à la pêche durant parfois plusieurs années. Ces tentatives, parfois maladroites, n'entament évidemment pas les monopoles des grands ports mais contribuent à accélérer la transformation de pêcheurs en marins. Des marins expérimentés qui feront alors l'impasse sur la pêche et pourront trouver plus simple d'embarquer sur un navire de commerce immatriculé à Nantes ou à Rouen. Incarnant ces évolutions, Pierre, Jean-Marie, Joseph-Marie et Hyacinthe Barbedienne seront, les uns ou les autres ou tour à tour, cultivateurs, graviers ${ }^{7}$, conditionneurs de morues, pêcheurs sur la côte de TerreNeuve, marins-pêcheurs " à Islande " et marins au commerce.

\section{De la terre à Terre-Neuve}

\section{Les Barbedienne, une famille de paysans bretons}

Pierre-Antoine Barbedienne naît à Pléneuf le 7 juin 1777. Il est fils de laboureur. Il se marie avec Adélaïde Boschet au début du XIX ${ }^{\mathrm{e}}$ siècle. Dix enfants naîtront de cette union; trois périront en bas âge. Resteront trois filles et quatre garçons dont le destin sera marqué, à une exception près, par la mer. Pierre-Antoine est cultivateur. Divers indices laissent à penser que la famille n'est pas pauvre malgré la très faible surface des terres qui lui appartiennent. Petit à petit, cependant, des difficultés apparaissent ${ }^{8}$ qui seront probablement à l'origine de la vocation maritime des Barbedienne.

En 1809, lors de la naissance de son fils aîné Pierre-Julien, Pierre-Antoine fait appel à deux cultivateurs de ses proches. Tous trois signent le registre, il n'y a pas de marin. Il n'y en aura pas davantage à l'occasion des naissances suivantes : Pierre-Antoine fréquente surtout les gens de son milieu, des laboureurs et quelques artisans ${ }^{9}$. Bien qu'à partir de 1815 l'activité maritime reprenne sur les côtes, les enfants Barbedienne auront une enfance de petits paysans probablement ignorants des choses de la mer. Aussi, lorsque son père meurt, en 1834, Pierre-Julien a-t-il une solide expérience

7. Le gravier est chargé de faire sécher les morues sur les graves (déformation de grèves) de Terre-Neuve.

8. En 1846, la veuve Adélaïde Boschet ne possède que 1,46 hectare. En outre, un seul bail de location de terre a été trouvé pour une "pièce de pâture " pour la période 18451854, Arch. dép. des Côtes-d'Armor : tables de l'enregistrement - 3P 191/6 et 3 Q 2164 et matrices du cadastre napoléonien de la commune de Pléneuf.

9. Pierre-Antoine a toutefois un neveu marin au commerce : Jean-Marie Guillois fils de sa sœur Jeanne. L'expérience avorte cependant rapidement puisque ce dernier, novice, déserte définitivement à Valparaiso à l'âge de dix-huit ans en 1835. Registre des novices du quartier de Saint-Brieuc ouvert en 1826. Service historique de la marine de Brest, $4 \mathrm{P} 386$. 
du travail de la terre. Naturellement, avec sa mère Adélaïde, il prend en charge l'exploitation familiale. En l'absence de son jeune frère Jean-Marie, il lui faut subvenir aux besoins de cinq frères et sœurs âgés de un à onze ans. La vie semble maintenant difficile et a peut-être obligé le frère cadet à quitter la demeure familiale. La famille, qui vit dans une petite demeure située dans le bourg et qui ne possède que très peu de terres, s'est probablement enfoncée dans la précarité. La vie suit son cours, rythmé par les travaux agricoles. Le temps passant, les jeunes frères et sœurs de Pierre participent de plus en plus, qui aux travaux domestiques, qui aux travaux des champs. D'ailleurs, lors du recensement de 1841, le jeune Joseph-Marie qui n'a que quinze ans est déclaré laboureur.

En 1838, Pierre Julien se marie avec Marie-Sainte Lévêque qui vient le rejoindre au domicile familial. De cette union naît en 1840, Pierre-Marie Barbedienne qui, devenu orphelin, sera alors mousse à la grande pêche. Deux ans plus tard, victime sans doute d'une de ces épidémies qui ravageaient alors les campagnes, Pierre-Julien meurt prématurément en octobre 1843. Marie-Sainte retourne alors avec son fils dans le village de la Ville Berneuf où demeurent ses parents. Quant à Adélaïde, elle doit s'occuper d'enfants encore jeunes. Il faut un homme à la maison. Ses fils aînés, JeanMarie et Joseph-Marie, sont en mer. Le plus jeune, Hyacinthe, n'a que dix ans. Une semaine après la mort de son frère Joseph-Marie débarque au Légué venant de Terre-Neuve mais, à dix huit ans, il est sans doute trop jeune pour prendre en charge l'exploitation familiale, et, de toute façon, il n'a pas fait son service militaire. Quant à Jean-Marie qui a vingt-six ans et est libéré de ses obligations militaires, il n'arrivera qu'en février 1844 car son navire est allé vendre sa cargaison de morues à Marseille. Avant de revenir au Légué, son bateau a fait route pour Le Havre pour livrer le vin, le savon et l'huile achetés à Marseille. Il est attendu pour succéder à son frère et s'occuper de la maison. Il lui faudra abandonner la navigation, s'installer dans l'exploitation familiale pour seconder sa mère et, à son tour, assurer le quotidien de ses jeunes frères et sœurs. Un chef de famille doit se marier. C'est chose faite deux mois après son retour. Mais, désormais, la mer est là. Il épouse une voisine, la sœur de son voisin François Barbedienne* avec qui il a déjà navigué vers la côte de Terre-Neuve. En outre, cette fois, les témoins de la cérémonie sont cultivateurs, artisans et marins. Ce mariage, d'une certaine manière, concrétise l'entrée officielle des Barbedienne dans le monde maritime.

\section{Quand un paysan devient laboureur de la mer}

Jean-Marie Barbedienne donc. C'est lui qui inaugure et qui confortera la vocation maritime de sa famille : il entraînera également frères, neveux et fils dans son sillage. Son acte de naissance, en 1817, porte la signature du notaire François Le Péchon. Un nom qui sera célèbre encore quarante ans 
plus tard dans le port de Dahouët et même au-delà ${ }^{10}$. Nous ne savons rien de son enfance sinon qu'il a appris à lire et à écrire. À treize ans, en principe l'âge du premier embarquement pour les mousses, il participe encore avec ses parents aux travaux agricoles. Ce n'est qu'en avril 1834 qu'il s'embarque pour la première fois : il a dix-sept ans, son père vient de mourir. Il va passer plusieurs mois dans un havre de la côte est de Terre-Neuve.

Que s'est-il passé? Pourquoi un embarquement si tardif? Avant la mort de Pierre-Antoine, le départ de Jean-Marie pour la mer aurait peut-être été inconcevable. Ce décès a sans doute été révélateur de difficultés rencontrées par la famille. Il semble clair maintenant qu'il faut des revenus complémentaires qu'il est difficile de trouver dans les exploitations agricoles voisines. Il est possible de chercher un embarquement dans le petit port naturel de Dahouët. Il est tout proche et, pour sa taille, très actif. Son activité, en tant que débouché de Lamballe, est essentiellement le cabotage et on y pratique aussi la petite pêche. Cependant, ce sont des milieux peu ouverts : les emplois s'y transmettent entre proches et, de toute façon, on y recrute peu. Par contre, de l'autre côté de la baie, on a besoin d'hommes pour aller à la pêche à Terre-Neuve. Depuis la fin des guerres napoléoniennes, les armateurs de Portrieux, Binic et du Légué ont reconstitué leurs flottes de bricks et de trois mâts. C'est une période prospère, les navires sont de plus en plus nombreux ${ }^{11}$ et on promet des gains substantiels. Comme autrefois, à partir de Dahouët, il est très facile de trouver une embarcation afin de rejoindre rapidement un proche ou un ami qui a été enrôlé au port du Légué. D'ailleurs, c'est ce que fait depuis plusieurs années le voisin François Barbedienne*. Et il n'est pas le seul à Pléneuf où la majorité des inscrits maritimes participe à la grande pêche à Terre-Neuve ${ }^{12}$.

Dorénavant donc, six mois sur douze, la vie de Jean-Marie sera radicalement différente de ce qu'il a connu. Tout change; paysages, climat, rythmes et conditions de travail. Il participe à la grande pêche. Grande, elle l'est sans doute par les distances, les flottes concernées, les quantités de morues pêchées. Cependant cette pêche-là, on l'oublie parfois, est sédentaire ${ }^{13}$; c'est une sorte de pêche côtière délocalisée. Les navires, bricks ou trois mâts, étant désarmés dans des baies abritées, au retour de la pêche, les hommes viennent à terre pour loger dans des baraques, certes rudimen-

10. François le Péchon est le père de Jean-Pierre qui sera d'abord notaire avant de se lancer ensuite dans l'armement.

11. Entre 1830 et 1844, le nombre de navires armés pour la grande pêche à Terre-Neuve passe de 33 à 40 au Légué et de 29 à 45 à Binic/Portrieux. Les jauges totales passent respectivement de 4135 à 5400 tonneaux et de 4400 à 6400 tonneaux. Service historique de la marine de Brest : rôles de désarmement - séries 4 P7 : 121 à 123/156 à 159 et 260 à 265.

12. Les registres matricule de l'Inscription maritime nous ont fourni les principales informations concernant les carrières des marins. Pour éviter des répétitions fastidieuses, nous fournissons la liste des séries consultées en annexe de l'article.

13. Contrairement à la pêche errante "à Islande " ou à la pêche à la ligne de fond sur les bancs de Terre-Neuve. 
taires, mais à l'abri des flots. Une partie de l'équipage n'embarque même pas dans les chaloupes qui partent en mer chaque jour et reste à terre pour traiter la morue. N'étaient-ce les conditions de travail parfois déplorables, on pourrait voir là la possibilité de faire doucement la transition entre terre et mer ${ }^{14}$.

Quant à Jean-Marie, on peut supposer que, comme d'autres mousses sans qualification, il est gravier c'est-à-dire qu'il doit étaler, retourner, surveiller les morues qui ont été étendues sur les grèves en vue de leur séchage. Un travail rude et peu considéré. En tout cas moins que celui des pêcheurs qui dans de petites embarcations partent chaque jour du rivage avec leurs sennes en quête de bancs de morues. Moins aussi que les décolleurs ${ }^{15}$, habilleurs et saleurs qui, sur les chauffauds ${ }^{16}$, vident et préparent les poissons avant de les confier aux graviers. Avec Jean-Marie, sur ce rivage d'une baie de Terre-Neuve, quelques dizaines d'hommes, amenés par le trois-mâts "Intimité " de l'armement Boullé, vivent sommairement dans des cabanes en bois. La répartition des tâches est précise, elle correspond à des revenus variés et, probablement, malgré une solidarité évidente, à des considérations différentes. À la fin de la campagne de pêche, en octobre, Jean-Marie est passé " en ressac ${ }^{17}$ " sur la côte de Terre-Neuve, il embarque sur le brick "Zéphir " du même armement ${ }^{18}$. Ce navire va livrer la morue en Méditerranée avec un équipage réduit de marins expérimentés tandis que l'« Intimité " ramène les autres au Légué. Jean-Marie est maintenant novice; il traverse l'océan pour la deuxième fois et, le 25 octobre 1834, il arrive à Gênes. Un port qui doit le surprendre lui, petit paysan qui n'était jusqu'alors pas sorti de son canton. Peut-être a-t-il l'autorisation de se rendre à terre ${ }^{19}$ pendant les négociations de vente. En effet, le navire reste là-bas un mois avant de repartir, chargé de diverses marchandises qu'il va livrer

14. LOTURE, Robert de, Histoire de la grande pêche à Terre-Neuve, Gallimard, Paris, 1949 256 p.; LACROIX, Louis, Les derniers Voiliers morutiers terre-neuvas, islandais, groenlandais, Luçon, 1949, 324 p.; MoRAndìre, Charles de la, Histoire de la pêche française dans l'Amérique septentrionale, 3 vol. éd. Maisonneuve et Larose 1962-1967. Ces travaux qui retracent l'histoire de la grande-pêche des origines à l'époque actuelle nous ont beaucoup aidé pour mieux cerner la vie quotidienne et les méthodes de pêche des terre-neuvas.

15. Sur les chauffauds de Terre-Neuve (voir note suivante), les décolleurs ouvraient les morues, ôtaient les foies et coupaient les têtes. Les habilleurs prenaient la suite des précédents : ils enlevaient les arêtes et jetaient les morues dans un traîneau.

16. Sorte de wharf surmonté en partie d'installations en bois où l'on préparait la morue débarquée à l'autre extrémité.

17. Il est donc transbordé d'un navire à un autre.

18. Les nombreux rôles de désarmement et leurs annexes (série P des archives de la marine de Brest et de Toulon; en ce qui concerne les archives départementales, série $7 \mathrm{R}$ à Nantes, $4 \mathrm{~S}$ à Rennes et $6 \mathrm{P}$ à Rouen) nous ont fourni les principaux renseignements concernant les navires, les campagnes, les cargaisons, les équipages, les spécialités des marins et les éventuels incidents. Comme en ce qui concerne les registres matricules, la liste des séries consultées est en annexe de l'article.

19. La lecture des rôles de désarmement montre que les restrictions au débarquement des équipages n'ont pas encore l'ampleur qu'elles prendront dans la seconde partie du siècle. On prenait également plus son temps durant les escales. 
à Saint-Malo. Il effectue ainsi une variante du trafic quadrangulaire que pratiquaient, à cette époque, les voiliers de la baie de Saint-Brieuc : dans les années trente, en effet, la plupart d'entre eux allaient de Terre-Neuve à Marseille livrer leurs morues séchées sur les graviers puis, chargés de savons, de vins et d'huile, ils allaient généralement livrer leur marchandise au Havre avant de revenir vers leurs ports d'attache ${ }^{20}$. Parfois, ils ramenaient du sel de Port-de-Bouc, de Hyères ou même des ports de l'Atlantique, pour la campagne suivante. En tout cas, pour Jean-Marie, c'est l'occasion de découvrir de nouveaux ports, de nouveaux paysages. Ce n'est plus un jeune paysan qui rentre à Pléneuf en janvier 1835, dix mois après son départ. Il a mûri, il a appris et découvert un monde nouveau dont, jusqu'à présent, il avait seulement entendu parler.

L'année suivante, en avril 1835, Jean-Marie s'embarque à nouveau au Légué pour Terre-Neuve. Sa seconde campagne se fait cette fois sur le brick " Alfred " de l'armement briochin de Kerautem. D'avril à octobre, il devient décolleur. Il doit donc être souvent sur le chauffaud occupé à sectionner les têtes des morues apportées par les pêcheurs. Il a moins souvent que les autres l'occasion d'aller dans les chaloupes que, de toute façon, il ne mène pas. Sans doute il exécute la fonction qui lui a été dévolue par les circonstances mais il n'est pas encore vraiment marin. D'ailleurs, le transbordement du produit de la pêche ayant été effectué sur un autre navire du même armement en route pour Marseille, Jean-Marie, cette fois, rentre directement au Légué avec une grande partie des équipages des deux navires. Comme lui, la plupart des mousses et novices sont à bien des égards restés terriens. Il n'est que de comparer les équipages pléthoriques des trois-mâts au départ du Légué ou de Binic (environ 60 hommes) avec ceux beaucoup plus modestes qui vont livrer la morue en Méditerranée (environ 15 hommes). Certains, comme Jean-Marie sans doute, se satisfont de cette situation qui les arrange dans un contexte de complémentarité familiale. D'autres, n'ayant parfois pas d'autres solutions, cherchent à s'embarquer pour le cabotage d'automne et d'hiver. À partir de maintenant donc, pendant six mois, Jean-Marie va seconder sa mère et son frère aux travaux des champs. Les deux années suivantes se passent de la même façon, partagées entre Terre-Neuve et Pléneuf. C'est ainsi qu'une modeste famille de paysans trouve le moyen de faire vivre une partie de ses membres.

Fin 1837, Jean-Marie a vingt ans. Il est inscrit maritime et est donc envoyé à la division de Brest avant d'embarquer sur des navires de la royale. Au bout de trois ans de formation et de navigation, il est libéré quartier-maître canonnier. Pour son milieu, c'est une promotion qui n'est pas négligeable. Surtout, il a acquis une spécialité qui lui donne quelques

20. En 1833, pour les armements de Binic, Portrieux et Le Légué, 87 \% des navires livrent leurs morues à Marseille et $63 \%$ de ces derniers vont ensuite au Havre. En 1836 on a respectivement $80 \%$ et $60 \%$, Service Historique de la Marine à Brest, rôles de désarmements : 4 P 7 127; 4 P 7 128; 4 P 7133 à 4 P 7135. 
compétences ${ }^{21}$. Lorsque, en avril 1842, il embarque, à nouveau au Légué, sur le brick "Boréal " de l'armement Sebert, il fait partie des matelots mieux rémunérés du fait de leur double spécialité. Il existe sur ce bateau un décolleur/charpentier, un décolleur/voilier, un senneur/calfat; JeanMarie, lui, est décolleur/calfat. Sa deuxième spécialité est, comme celles des précédents, éminemment utile sur un navire. Après la campagne de pêche, Jean-Marie fait partie des marins, peu nombreux, qui sont envoyés à Marseille livrer directement la morue sèche. De retour le $1^{\text {er }}$ février 1843 à Saint-Brieuc, il réembarque presque immédiatement sur un brick armé au cabotage expédié pour aller chercher, à l'île de Ré, le sel nécessaire aux campagnes de pêche. Il débarque au Légué le 14 avril et, le 27 du même mois, il s'embarque sur le trois-mâts " Saint Louis ", commandé par le capitaine Pierre Desury ${ }^{22}$ pour un long périple quadrangulaire. Ce n'est qu'en février de l'année suivante, qu'il est de retour dans sa famille. On le voit, Jean-Marie est maintenant un vrai marin à temps complet qui n'a plus la possibilité de participer aux travaux agricoles. Donc, lorsqu'il arrive à Saint-Brieuc en février 1844, il a de nouveau fait une campagne complète en passant par Terre-Neuve Marseille et Le Havre. Son avenir semble tracé : il est sur la mer. Cependant, à peine débarqué, il apprend le décès de son frère aîné. On compte sur lui à la ferme. Alors, peut-être à contre cœur, il s'installe au pays pour remplacer Pierre-Julien et s'occuper de la terre. Ce n'est probablement pas un hasard si, comme nous l'avons vu, il se marie aussitôt. Deux ans plus tard, Jean-Marie et Jeanne sont installés dans la maison voisine, chez la propriétaire, la tante de Jeanne et de son frère François. Jean-Marie a-t-il la nostalgie de la mer? Envie-t-il son beau-frère François Barbedienne* qui, chaque année, va faire six mois de campagne à Terre-Neuve?

\section{Quand un pêcheur devient marin-pêcheur}

Jean-Marie attend probablement une occasion pour reprendre la mer. Elle se présente rapidement. En effet, vers la fin des années quarante, un notaire et un négociant de Pléneuf se lancent dans l'armement à la grande pêche et recrutent des équipages : ce sont Rubin de Rays et Jean Pierre Le Péchon. Plus tard, d'autres les imiteront et se lanceront dans la même activité, en particulier Carfantan et Boutevillain de Grandpré. Ainsi, alors qu'au début de la décennie, aucun navire ne quitte Dahouët pour la grande pêche, à la fin, il y en aura sept totalisant 872 tonneaux de jauge. C'est en pleine conscience que les édiles accompagnent et favorisent cette expansion qui stimule l'économie locale et provoque l'augmen-

21. Sur les qualifications des inscrits maritimes et les écoles de spécialités de la marine voir LE BOUËDEC, Gérard, Bretons sur les mers, Rennes, Ouest-France, 1999, 251 p., voir p. 230 et 231 .

22. Auteur d'une fort instructive description de campagnes de pêche. $C f$. Pierre-Romain Désury, "A bord d'un Terre-Neuvas de Saint-Brieuc vers 1840 ", Le Chasse-marée, n 123, p. 18 à 29 . 
tation de la population ${ }^{23}$. Ainsi, en 1851, la municipalité déclare l'armement local source de "bien être " pour la commune même si, par la suite, pour obtenir l'implantation d'une brigade de gendarmerie, elle se plaint auprès du préfet de l'insécurité provoquée par l'afflux de populations extérieures ${ }^{24}$.

La création d'armements à Pléneuf coïncide avec d'autres événements extérieurs au monde maritime. Dans cette commune, comme ailleurs en Bretagne, à la suite d'un hiver extrêmement rigoureux, la moisson de 1846 est catastrophique et le mildiou continue à ravager les pommes de terre ${ }^{25}$. L'hiver suivant est tout aussi calamiteux que le précédent. Ce n'est donc probablement pas un hasard si Jean-Marie reprend la mer au printemps 1847. Par ailleurs, ses frères et sœurs ont grandi le libérant de certaines contraintes. Il quitte donc la demeure familiale, s'installe chez sa femme et son beau-frère et, en avril 1847, en compagnie de ce dernier, il embarque sur le brick "Saint Brieuc " en qualité de calfat. Alors que son beau-frère est passé, comme habituellement, en ressac, Jean-Marie fait la campagne complète et arrive à Dahouet en mars 1848 après un long voyage qui l'a fait passer par Marseille, Dunkerque, Sunderland et Le Croisic. Pour la première fois, il est le témoin direct des dangers du métier : il voit, lors d'une tempête, Stanislas Barbedienne* tomber du grand hunier et périr en mer. En avril il embarque sur le même navire accompagné cette fois de ses deux jeunes frères. Il y a Joseph-Marie qui, rentré de son service militaire, profite lui aussi des facilités procurées par un armement local ainsi que le plus jeune Hyacinthe qui embarque pour la première fois comme mousse. Les problèmes de l'agriculture ont sans doute poussé le jeune garçon à faire un choix que ses aînés ont encouragé. Jusqu'en 1853, Jean-Marie fait les campagnes de pêche avec Joseph-Marie toujours sur le "Saint Brieuc " de l'armateur Rubin de Rays, commandé par le capitaine Jean François Hamonet. Calfat confirmé, il embarque à la fin de chaque campagne à Terre-Neuve pour les ports de Méditerranée. Une routine s'installe quoique, progressivement, les choses changent.

En effet, curieusement, la création des armements à Pléneuf un peu avant le milieu du siècle, s'est effectuée au moment où les difficultés augmentent à Terre-Neuve pour les autres armements de la baie de Saint-Brieuc ${ }^{26}$. De plus, les frets traditionnels de savon de Marseille pour Le Havre se raréfient : après déchargement de la morue, de nombreux navires doivent se

23. 1816 habitants au recensement de 1836 et 2200 en 1866, Arch. dép. des Côtes d'Armor, 6 M 391 et 6 M 392.

24. Guigot, André, Dahouët, port de Bretagne. Tome 1. Saint-Brieuc : Breizh-Compo, 1988, p. 71-124 et Dahouët, port de Bretagne. Tome 2. Saint-Brieuc : Breizh-Compo, 1990, p. $105,136$.

25. BREKILIEN, Yann, Les Paysans bretons, Paris, Hachette, 1966, p. 18-19.

26. Les colons britanniques sont de plus en plus nombreux, les tensions augmentent, certains havres sont contestés. À la fin des années quarante, les tonnages sont à la baisse. LOTURE, Robert de, op. cit., p. 94; La MORANDIÈre, Charles de, op. cit., p. 1186-1187. 
contenter, pour le retour, du sel de Port de Bouc ou d'Hyères ${ }^{27}$. Quand, plus tard, les terre-neuviers sont réarmés à Marseille, il n'est pas rare que ce soit pour le long cours. Bien sûr, il reste encore l'huile, le vin, le noir animal ${ }^{28}$ que l'on embarque pour Rouen ou Dunkerque, mais les routes habituelles ne reprendront pas. Le commerce, inéluctablement, se diversifie et les trajets de retour se font progressivement plus complexes et plus variables. Les armateurs doivent se tenir prêts à toute opportunité, à saisir de nouveaux marchés. D'ailleurs, la révolution industrielle ouvre de nouvelles perspectives. Ainsi, tel voyage permet de transporter des rails de Gênes à Cardiff et de revenir avec une cargaison de charbon pour Brest et Le Havre. Lors de tel autre, un trois-mâts au retour de Marseille prend une cargaison de minerai de manganèse dans un port espagnol, va le livrer à Dunkerque puis va chercher à Swansea une cargaison de charbon pour la livrer au Havre, repart dans le même port reprendre du charbon, cette fois pour Nantes et revient enfin au Légué en mars pour la nouvelle campagne de pêche. Il s'agit de rentabiliser au maximum des bâtiments qui doivent rester le moins possible désarmés. L'automne et l'hiver, le cabotage a tendance à se prolonger tandis que la liste des ports visités s'allonge. L'horizon de Jean-Marie s'élargit au fur et à mesure que son expérience s'accroît.

En septembre 1853, toujours fidèle à l'armement de Rays, Jean-Marie embarque sur le brick " Marie Victoire " ou la goélette "Saint Prosper ", avec le capitaine Marie-Ange Lesage. Maintenant, il ne s'absente plus une année entière : les trajets directs Terre-Neuve Marseille se font plus rares. Chaque année, en octobre, au retour de Terre-Neuve, il débarque d'abord à Dahouët avec les pêcheurs, ce qui lui permet de rendre visite aux siens. Peu de temps après, le même voilier est réarmé pour le cabotage et JeanMarie embarque à nouveau. On va toujours chercher du sel à l'île de Ré ou au Croisic ou livrer la morue dans les ports de Méditerranée mais le commerce du charbon prend de plus en plus d'importance. Les voiliers vont maintenant régulièrement à Cardiff ou à Swansea prendre des cargaisons de charbon pour quelque port de l'Ouest de la France. On profite de ces voyages pour livrer du froment et des pommes de terre en Grande Bretagne. On va également en Suède chercher du bois et du minerai de fer. Jean-Marie, qui était devenu maître calfat en avril 1854 devient second en octobre. Il est maintenant définitivement officier ${ }^{29}$ et il arrive au sommet de sa carrière puisqu'il ne sera jamais capitaine ${ }^{30}$. Son expérience lui donne de l'impor-

27. En 1850, sur les 17 morutiers du Légué et de Dahouët allés vendre leurs morues en Méditerranée, 11 reviennent avec une cargaison de sel, 3 seulement vont au Havre avec " divers matériels ", Service historique de la Marine, Brest 4 P 7175 et 4 P 7176.

28. Engrais constitué des résidus des sucreries nantaises qui employaient de la poudre d'os pour filtrer la mélasse.

29. Durant la première partie du siècle, à la grande pêche, le titre ne correspond à aucun diplôme; il est le fruit de l'expérience et des relations avec l'armement. Il en était souvent de même pour les capitaines pour lesquels un examen n'a été imposé qu'à partir du décret de 1852.

30. Pour connaître les conditions permettant d'être maître de cabotage voir : LE BOUËDEC, Gérard, Bretons sur les mers, op. cit., p. 198. 
tance ce qui lui permet d'amener avec lui Pierre-Marie ${ }^{31}$, son neveu qui, à 15 ans, est embarqué comme mousse : ils navigueront ensemble jusqu'au service militaire de ce dernier en 1860. Pourtant, à son retour, en 1863, Pierre-Marie ne reprend pas la mer. Il l'avait annoncé par deux fois, durant son service militaire, en 1861, au commandant de son navire ${ }^{32}$. C'est donc une décision mûrement réfléchie. A-t-il été déçu? A-t-on besoin de lui? Quoi qu'il en soit, son oncle ne l'aura pas convaincu : il rejoint son beau-père et sa mère au village de Trémensel en Pléneuf.

À partir d'avril 1861, Jean-Marie navigue, cette fois, en compagnie de son fils Jean-François. Ce dernier a treize ans, l'âge d'être mousse. Est-il volontaire? Son père l'a-t-il poussé, montrant ainsi l'importance qu'il accorde à la carrière maritime. En tout cas, le jeune homme fait rapidement l'expérience des aléas de la profession En janvier 1862, la goélette " Diane " sur laquelle son père et lui sont embarqués coule près de la côte sud de l'Angleterre. Ils sont rescapés et ne semblent pas trop affectés puisque, après leur rapatriement à Saint Malo, ils s'embarquent à nouveau pour Terre-Neuve. Un changement toutefois; ils naviguent désormais sur les navires d'un autre armateur de Pléneuf : Jean-Pierre Le Péchon. La malchance cependant poursuit le jeune mousse : la goélette "Philoména " sur laquelle son père et lui sont embarqués fait naufrage le 25 janvier 1863 à la pointe d'Erquy. Il n’y a pas, certes, de pertes humaines mais c'en est trop. Jean François n'a pas envie de prolonger l'expérience; il ne réembarque pas et, au recensement de 1866, il se déclare menuisier puis, à la fin de l'année, il déclare renoncer à la navigation et est rayé des matricules de l'inscription maritime. JeanMarie est-il sûr de son choix ou bien les circonstances l'y incitent-elles? Au demeurant, il persiste dans le prosélytisme : lors de la campagne de 1864 il fait embarquer avec lui un autre neveu, Jean François Barbedienne ${ }^{* 33}$, qui vient également d'avoir treize ans. Mais ce dernier, fils d'agriculteurs, ne fait qu'une seule campagne à Terre-Neuve : comme son cousin, il retourne définitivement au village et ne réembarque pas.

\section{Une dernière campagne longue et symbolique}

Quand Jean-Marie embarque pour le dernière fois, le 28 avril 1865 sur le « Reine des anges " de l'armateur Jean Pierre Le Péchon il est encore une fois second. Le navire est commandé par le capitaine Mathurin Ancelin. Savent- ils l'un et l'autre que la campagne sera aussi longue? En tout cas, elle sera le couronnement de la carrière de Jean-Marie par sa durée et par la variété des ports visités. Ce sera également une fin de carrière prémonitoire de l'évolution des situations professionnelles des frères Barbedienne. En

31. C'est le fils unique du défunt Pierre-Julien. Il vivait avec sa mère dans le village d'origine de cette dernière.

32. Registre matricule, Quartier de Saint-Brieuc, Arch. dép. des Cotes d'Armor, 4 P 3120.

33. C'est le fils de son beau-frère le laboureur Pierre Barbedienne* époux de sa soeur Marie-Jeanne. 
effet, pour une campagne de vingt-huit mois, à peine six auront été consacrés à la pêche à Terre-Neuve : Jean-Marie termine donc comme un marin au commerce. C'est une fin et un tournant concrétisés en outre par l'embarquement en 1866 de son jeune frère Hyacinthe qui, lui, navigue au commerce depuis longtemps. Une campagne en apothéose pour les Barbedienne puisque c'est durant celle-ci que Hyacinthe deviendra lieutenant sur le même navire. Jean-Marie passe la main au moment où il rejoint son cadet.

Étonnante campagne en effet que la dernière de Jean-Marie. Elle commence de manière classique par la pêche à Terre-Neuve, la livraison de la morue à Marseille et le retour à Rouen pour y livrer des marchandises méditerranéennes. À partir de ce moment, le navire armé au cabotage ${ }^{34}$, va se consacrer uniquement au commerce. En mars 1866, il est expédié à Messine puis va chercher une cargaison de soufre à Agrigente pour la livrer à Dunkerque. Là-bas, il remplace le soufre par du charbon qu'il va décharger à Brest. Il est alors envoyé sur lest à Swansea pour y renouveler sa cargaison de charbon et la livrer à Alger. De là, il va à Trieste prendre une cargaison de douelles qu'il va livrer à Nantes où il est désarmé en août 1867. Le « Reine des anges " n'est plus un morutier. Il pratique un cabotage d'opportunité.

Comme d'autres armateurs de la baie de Saint-Brieuc, en effet, Le Péchon, face aux difficultés de la grande pêche, réoriente partiellement ses activités. Non seulement, comme nous l'avons vu, les navires de pêche sont de plus en plus intensément utilisés pour le commerce durant l'automne et l'hiver, après la saison de pêche, mais en plus, progressivement, certaines unités y sont maintenant consacrées totalement. Ceci, d'autant que les opportunités ne manquent pas. Bien sûr, le petit cabotage commence à subir les conséquences fâcheuses du développement du chemin de fer mais le grand cabotage, entre Manche-Atlantique et Méditerranée, entre la Grande Bretagne et la France, là où la concurrence du rail ne se fait pas sentir, ne fait que progresser. C'est que le développement de l'industrie suscite de nouveaux besoins et les armateurs, morutiers ou non, savent en tirer profit.

Après une longue carrière de terre-neuvas, Jean-Marie termine donc sa vie active, en août 1867, de manière surprenante, sur un terre-neuvier devenu grand caboteur. Il a alors cinquante ans et peut faire valoir ses droits à la retraite. Avant de se retirer, il lui reste toutefois une dernière formalité. Pour justifier de cinq ans de service à l'état, il va passer deux semaines sur un cutter $^{35}$ de la marine de guerre comme canonnier. Il se retire alors dans

34. Pour les définitions du petit et du grand cabotage voir : LE BouËDEC, Gérard, Bretons sur les mers, op. cit., p. 178. Cette campagne de la "Reine des anges " sera d'ailleurs marquée par des irrégularités dans le type d'armement. Elles ont été relevées dans une lettre annexée au rôle de désarmement envoyée par le commissaire à l'Inscription maritime de Saint-Brieuc à son collègue de Nantes, Arch. dép. de Loire Atlantique, 7 R 4558.

35 . Terme anglais à peu près synonyme de cotre. Utilisé parfois au XIX ${ }^{\mathrm{e}}$ siècle pour les cotres rapides de la marine royale. Ici, le terme est extrait des registres matricules. 
sa maison du bourg de Pléneuf avec sa femme, son fils menuisier et sa fille qui épousera plus tard un autre marin. Il meurt le 18 décembre 1871 n'ayant profité que de quatre ans de retraite.

\section{De Terre-Neuve à Islande}

\section{Une option qui s'ancre}

Joseph Marie Barbedienne, le second marin de la fratrie, est né à Pléneuf en 1825. Après avoir fréquenté l'école comme son frère aîné, il commence, lui aussi, sa carrière maritime tardivement. En effet, lors du recensement de 1841, il est encore présenté comme laboureur. Ce n'est qu'à dix-sept ans, en avril 1842, qu'il embarque comme novice, au Légué, sur le brick " Triton " de l'armement de Villeféron. Il va lui aussi à Terre-Neuve. Là-bas, comme il n'a aucune compétence particulière, on l'affecte à un poste de traineur ${ }^{36}$ sur un chauffaud. Contrairement à son frère, il a très vite, l'expérience concrète des dangers du métier : en juillet il est témoin du naufrage d'une chaloupe et de la mort de ses trois occupants. Dure expérience qui se renouvelle l'année suivante : une autre chaloupe de pêche disparaît avec son équipage. Joseph-Marie est cette fois banquier ${ }^{37}$. L'année suivante, il est décolleur. On le voit, Joseph Marie n'apprend pas encore le métier de marin mais celui de pêcheur. D'ailleurs, il ne fait que les campagnes de pêche d'avril à octobre. Cependant, il acquiert une expérience de plus en plus large des techniques de la pêche et de la préparation de la morue. Une expérience précieuse pour des employeurs qui, à l'époque, considèrent parfois le capitaine avant tout comme un maître de pêche. Il apprend donc son métier tout en fournissant aux siens quelques revenus supplémentaires. Bien sûr, le reste du temps, maintenant qu'il est adulte, il demeure, à Pléneuf, en l'absence de Jean-Marie, un élément important de l'exploitation familiale. Toutefois, son avenir n'est pas encore tracé : il faut, au préalable, régler le problème du service militaire. Son grand frère Jean-Marie vient de revenir à la terre. C'est l'occasion pour lui de partir. En novembre 1844, Joseph-Marie est levé par anticipation, sur sa demande. Il fait trois années de service dans la royale et est libéré en février 1847.

\section{Un moment de pluriactivité}

À son retour, les choses ont changé. Marie-Jeanne, sa sœur aînée s'est mariée avec un cultivateur : toute la famille est partie avec eux au village de la Motte. Son frère aîné, Jean-Marie, est également marié, il demeure au bourg et est installé définitivement dans la carrière maritime. Joseph-Marie

36. Le traîneur est un novice ou un mousse qui doit tirer un traîneau chargé de morues étêtées et vidées pour le porter aux saleurs.

37. Le banquier est généralement un mousse ou un jeune matelot embarqué dans une chaloupe de pêche : il est chargé particulièrement du cordage situé à l'avant et de jeter le grappin sur les lieux de pêche. 
ne peut que revenir habiter avec sa mère et ses autres frères et sœurs pour remplacer son grand frère. Mais, depuis son départ, les choses ne se sont pas arrangées, bien au contraire. La terre ne nourrit plus les Barbedienne, il faut absolument d'autres revenus. On convient donc d'une demi-mesure : comme avant son service militaire, d'octobre à avril, Joseph-Marie travaillera à la terre et le reste du temps, il ira gagner un peu d'argent à TerreNeuve qu'il connaît bien. Pas de problème, son grand frère Jean-Marie lui présente les avantages et les facilités apportés par les nouveaux armements locaux. Il se saisit de cette opportunité : les deux embarquent en avril 1848 sur le brick Saint-Brieuc de l'armateur Rubin Derays pour les havres de Terre-Neuve. En outre, ils emmènent avec eux leur jeune frère Hyacinthe qui va avoir quinze ans.

Durant cinq ans, de 1848 à 1852, Joseph-Marie fait toutes les campagnes de pêche à Terre-Neuve. Il embarque toujours sur le même brick commandé par le capitaine Jean François Hamonet. Il accompagne son frère Jean-Marie mais ne le suit pas durant les campagnes de cabotage. À Terre-Neuve, il est maintenant senneur et complète son expérience de la pêche. À la fin de chaque saison, il passe en ressac sur le brick Marie-Victoire du même armateur et débarque à Dahouët en octobre. Il reste alors avec les siens demeurés à terre, comme avant son service militaire. Joseph-Marie est un paysan-marin ou plutôt un paysan-pêcheur.

\section{Retour à la mer}

Cependant, sa situation va évoluer. Les années suivantes seront en effet l'occasion d'un tournant dans la carrière de Joseph-Marie. Déjà, en 1851, en compagnie de son frère aîné, il avait fait une campagne complète. Il avait sans doute été symboliquement admis dans le corps des vrais marins à l'occasion de son premier passage au large du "Mont aux singes ${ }^{38}$ ". Il était allé ensuite à Gênes, Cette ${ }^{39}$ et Rouen avant de rentrer à Dahouët.

Mais, c'est à partir de 1853 que les choses changent pour de bon. Les revenus de la mer s'avèrent sans doute définitivement plus avantageux pour la famille. Joseph-Marie embarque à nouveau sur le Saint-Brieuc. Le navire est commandé par Joseph Conan. Joseph-Marie est maintenant, sans doute, considéré comme un marin suffisamment expérimenté et fiable. En tout cas, son capitaine le prend dans son équipage pour aller en Méditerranée. Après Porto et Marseille, le navire est expédié au cabotage d'abord au Havre puis à Cardiff et à Nantes. La campagne 1854-1855 est l'occasion d'un autre périple qui mène Joseph Marie, après Terre-Neuve à La Rochelle, à Marseille puis à Alexandrie. Par l'intermédiaire du capitaine, son armateur le sollicite déjà, lui et d'autres matelots, pour la prochaine

38. C'est le rocher de Gibraltar. D'après le capitaine Desury, le passage du détroit est l'occasion d'un baptême pour les néophytes et de réjouissances pour l'ensemble de l'équipage, Le chasse-Marée, art. cit. n 123, p.18 à 29.

39. Graphie de l'époque pour Sète. 
campagne. Le capitaine est manifestement satisfait d'un équipage qu'il connaît bien, avec lequel il forme une équipe solidaire ${ }^{40}$ fiable et professionnelle. Malheureusement pour lui, c'est la guerre de Crimée et il redoute le passage au bureau de l'inscription maritime. Lui-même et son armateur tentent de soustraire au recrutement leurs meilleurs marins, mais les célibataires comme Joseph-Marie ont peu de chance d'y échapper. Pourtant, en soudoyant un fonctionnaire du port de Marseille, Joseph Conan parvient à garder tous ses hommes pour faire son voyage à Alexandrie ${ }^{41}$. À son retour, il n'est plus possible de soustraire Joseph-Marie au recrutement. En juillet 1865, Joseph-Marie quitte le Saint-Brieuc et se dirige immédiatement sur Toulon où il est levé.

Aussitôt, Joseph-Marie embarque comme matelot sur le Neptune de la marine impériale. Il participe aux combats de la mer noire et est libéré un an plus tard, en août 1856, avec une médaille. Sa vocation maritime s'est peut-être consolidée : la terre s'éloigne de plus en plus. Dès son retour, après un court embarquement au bornage, il va à Marseille s'embarquer à nouveau sur le Saint-Brieuc. Le brick est cette fois armé pour un long voyage commercial de quatorze mois qui le mènera, entre autres, à La Plata. Un pas de plus est franchi par Joseph-Marie qui traverse pour la première fois l'équateur. Pour des raisons qui nous échappent il ne réitérera pas l'expérience. En effet, en avril 1858, il reprend la grande pêche; d'abord sur la goélette Saint-Prosper puis sur le brick Marie-Victoire du même armement. À Terre-Neuve, son professionnalisme s'affirme également : il est maintenant " avant de bateau " et participe activement aux manœuvres des grosses chaloupes de pêche. Comme avant la guerre, il alterne pêche à Terre-Neuve et campagnes de cabotage. Comme son frère aîné, il est rarement à Pléneuf avant le mois de février. Joseph-Marie est devenu lui aussi un marin-pêcheur.

\section{Pêcheurs d'Islande, pêcheurs nomades}

En 1860, Joseph-Marie décide de se marier. Il a maintenant trente-cinq ans et un métier sûr. En outre, ses deux jeunes sœurs, Sainte et Adélaïde, se sont elles-mêmes déjà mariées. La plus âgée, restée à la terre, a pris en charge sa mère, la vieille Adélaïde qui a plus de soixante quinze ans. Quant à son jeune frère, Hyacinthe, c'est déjà un marin du commerce confirmé, qui est d'ailleurs en route pour le Vénézuéla. Joseph-Marie, libre de toute responsabilité, épouse une voisine Joséphine Audrain. Les quatre témoins de la cérémonie sont, cette fois, tous marins : la famille Barbedienne a basculé définitivement du côté de la mer. Elle fréquente maintenant beaucoup les marins. Il n'y a évidemment aucune difficulté à se faire de telles relations dans une commune où il $\mathrm{y}$ a une centaine de marins en activité pour une

40. Tous les hommes de l'équipage sont de Pléneuf ou des environs immédiats.

41. Informations tirées de la correspondance entre le capitaine Joseph Conan et son armateur Rubin Derays, Archives municipales de Saint-Brieuc, 10 Z 5. 
population totale d'un peu plus de deux milles habitants ${ }^{42}$. Ainsi, par son mariage, Joseph Marie devient le beau-frère d'un de ses amis, Mathurin Lévêque, terre-neuvas comme lui, qui a épousé l'autre fille Audrain. Les deux couples s'installent ensemble chez la mère des deux filles. JosephMarie et Joséphine auront quatre enfants. Seul, le premier des fils, JosephLéon, reprendra le flambeau maritime. Mais, c'est dans la marine de commerce qu'il fera carrière, initié en cela par son oncle Hyacinthe, qui le fera embarquer comme mousse à Saint-Nazaire.

Le mariage de Joseph-Marie coïncide avec une modification importante dans sa carrière de marin. À partir de 1860, en effet, les choses changent dans les armements de la baie de Saint-Brieuc. Des difficultés se manifestent à Terre-Neuve. D'abord, la morue devient plus rare dans les zones côtières fréquentées par les pêcheurs. Il y a également des difficultés croissantes avec les habitants de Terre-Neuve et les autorités britanniques. En effet, la pêche traditionnelle, côtière et sédentaire nécessite la construction à terre d'installations permanentes autorisées, certes, par un traité conclu entre la France et l'Angleterre mais qui provoquent le mécontentement des immigrants britanniques de plus en plus nombreux. Les autorités de Londres réagissent mollement aux agissements de ces derniers qui vont jusqu'à envisager leur rattachement aux Etats-Unis. Face à cette situation, les armateurs vont utiliser diverses stratégies. Certains, comme nous l'avons vu avec Le Péchon à Dahouët, augmentent la part de leur activité au cabotage. Au Légué, certains armateurs, tel de Villeféron, envoient deux ou trois navires pêcher sur les grands bancs ${ }^{43}$ au large de Terre-Neuve en utilisant Saint-Pierre et Miquelon comme base arrière. Cependant, au Légué, on restera encore longtemps attaché à la tradition de la grande pêche sédentaire. Ailleurs, dans les autres ports de la baie de Saint-Brieuc, le changement sera plus sensible.

Après Dunkerque, Paimpol arme en 1852 une goélette pour l'Islande. Tandis qu'à Saint-Brieuc on reste fidèle aux havres de Terre-Neuve, à Binic et à Portrieux on suit l'exemple paimpolais. Dans ces deux derniers ports, la proportion des navires armés pour l'Islande ne cesse d'augmenter. Progressivement, les armateurs de Pléneuf se lancent à leur tour dans ce nouveau type de grande pêche. En 1858, Prosper Carfantan expédie le lougre " Alexandre "; de Rays, Le Péchon, Boutevillain de Grandpré suivent ${ }^{44}$. Après quelques tâtonnements, c'est le succès. Le trajet est en effet plus court : à mi-campagne, en juin-juillet des chasseurs peuvent aller chercher le produit de la première pêche c'est-à-dire la morue "verte " qui, salée

42. D’après les recensements de Pléneuf 1851 et 1856, Arch. dép. des Côtes-d'Armor, 6 M 391 et 6 M 392.

43. Dans ce type de pêche qui ne concerne pas nos personnages, de petites embarcations partent d'un grand voilier mouillé sur les bancs, au large de Terre-Neuve, pour placer ou relever des lignes de fond.

44. En 1860, à Dahouët, les registres et les rôles de désarmement signalent 11 navires armés pour la grande pêche : 9 qui totalisent 746 tonneaux de jauge vont à Islande tandis qu'il n'y en a plus que 2 totalisant 310 tonneaux qui vont sur la côte de Terre-Neuve. 
mais non séchée, s'écoule à meilleur prix que la morue sèche. Par contre les conditions sont, comme sur les bancs de Terre-Neuve, beaucoup plus rudes pour des marins qui, toujours embarqués, subissent sans discontinuer les dangers d'une mer souvent agitée, la promiscuité, des conditions de vie peu hygiéniques. Certes, les Islandais reviennent un peu plus tôt que les terre-neuvas ${ }^{45}$ mais le départ a tendance à se faire à une date de plus en plus précoce ${ }^{46}$. Quant à la méthode de pêche, elle est tout à fait différente de celle pratiquée à Terre-Neuve : pendant que le navire se laisse dériver au large des côtes d'Islande, les hommes, alignés sur un bord, pêchent avec des lignes face au vent. En principe, les goélettes ne vont relâcher dans un fjord abrité qu'une fois au cours de la saison, le reste du temps, elles sont en mer. C'est une différence notable avec la pêche sédentaire. Ce dur régime ne peut qu'accélérer l'expérience maritime des hommes même si par ailleurs tout l'équipage sans exception doit participer à pêche. Plus personne ne travaille à terre et pour les jeunes ruraux, il n'y a plus dans ce type de pêche de transition possible entre terre et mer. En outre, alors que nous avons noté qu'un terre-neuvas sur quatre prolongeait la campagne sur son navire, entre un tiers et la moitié des Islandais font de même. Quoi qu'il en soit, certains marins-pêcheurs, par obligation ou par curiosité vont se laisser convaincre par cette nouvelle " épopée " tandis que d'autres, les plus âgés sans doute, conservent leurs habitudes anciennes. De jeunes ruraux iront tout de même chercher "à Islande " une issue à leur pauvreté mais le choc sera rude et le choix définitif plus rapide qu'auparavant.

Donc, fin mars 1860, Joseph-Marie, quinze ans avant de se retirer, entame une nouvelle carrière : il embarque sur le lougre ${ }^{47}$ " Charles Louis " de l'armateur Boutevillain de Grandpré : destination les fjords d'Islande. La campagne terminée, le navire arrive à Dahouët le 16 août avec une cargaison de morues : le navire est immédiatement réarmé pour le cabotage et, avec six hommes d'équipage sur les seize précédemment embarqués, il repart pour un voyage qui l'amènera à La Rochelle, Dunkerque et Cadix. Joseph-Marie, qui est maintenant un vrai marin est, bien sûr, du nombre des matelots embarqués pour le cabotage. Il en est de même l'année suivante. Lorsque, en 1862 et 1863, il s'embarque sur la bisquine "Saint François » de l'armement Chauvin et Cie, les choses se passent de la même façon : retour fin août-début septembre à Dahouët, réarmements au cabotage pour la livraison de la morue dans un port de l'Atlantique puis, cabotage jusqu'au mois de janvier suivant. Quant aux campagnes de cabotage, on l'a vu, elles sont à cette époque de plus en plus variées tant en ce qui concerne les matières transportées que les escales fréquentées. En 1864, Joseph-Marie, déjà islandais expérimenté, est nommé second comme son frère aîné.

45. Août-septembre plutôt que septembre-octobre.

46. Chappé, François, L'Épopée islandaise 1830-1914, Paimpol, la République et la mer, Thonon-les-bains, édition l'Albaron, 1990, 382 p.

47. Au début, on expédiait à Islande des lougres, des chasse-marées et des bisquines; par la suite, les goélettes s'imposent comme les bâtiments les mieux adaptés. 
De 1865 à 1867, à nouveau sur le lougre « Charles Louis » de Boutevillain de Grandpré, il fait les campagnes de pêche et de cabotage avec un proche de la famille, le capitaine François Lévêque. Joseph-Marie, en tant que second, est confronté à la tragique disparition de ce dernier au large de l'Islande en juin 1867. À partir de 1868, Joseph Marie, toujours officier, navigue sur la goélette "Saint Guillaume » de Jean Pierre Le Péchon : Islande et cabotage se succèdent jusqu'en 1872 .

\section{Un moment de flottement}

Alors que la fin de sa carrière semble inéluctablement tracée, JosephMarie termine son itinéraire de façon surprenante : il semble hésiter sur la voie à suivre. S'en suivent quelques problèmes. Mais peut-être des difficultés professionnelles sont-elles à l'origine de cette fin de carrière inattendue?

En 1872, il réembarque une dernière fois pour Terre-Neuve avant ses deux dernières campagnes islandaises. Nostalgie, incertitude ou problèmes avec les armateurs? Cette année-là, il ne réembarque pas au cabotage et, lors du recensement, se déclare, pour la première et unique fois, marchand. En outre, il n'est plus officier mais maître d'équipage. Il a décidé de prolonger considérablement ses séjours à terre. Il passe maintenant l'automne et l'hiver au foyer. Peut-être est-il las et aspire-t-il à la retraite ou, tout au moins, à gérer le petit commerce ${ }^{48}$ dont s'occupe son épouse Joséphine? Il décide toutefois d'aller jusqu'au bout. Mais de fâcheux incidents gâchent ses deux dernières campagnes islandaises : une mutinerie en 1873 alors qu'il est maître d'équipage ${ }^{49}$, deux noyades en 1874 . De toute façon, Joseph-Marie arrive en fin de carrière. Fin 1874, il s'embarque une dernière fois au bornage ${ }^{50}$ jusqu'au 28 février 1875; il a alors cinquante ans, il peut se retirer dans son foyer et s'occuper de sa boutique. Jusqu'au début du siècle suivant, Joseph-Marie et son épouse conserveront ce commerce au bourg. C'est en 1910, à 85 ans, que Joseph-Marie, ayant profité d'une longue retraite, meurt à Pléneuf.

\section{Le monde comme horizon}

\section{Le passage à la marine de commerce}

Hyacinthe Barbedienne, le dernier des Barbedienne, est né à Pléneuf en 1833. Il ne connaîtra pas son père qui décède l'année suivante. Il subit

48. Information provenant du recensement.

49. Le rapport du capitaine de frégate commandant la station d'Islande (conservé en apostille dans un rôle de désarmement) n'est " pas favorable au capitaine " de la goélette. Rôle de désarmement de la goélette "Maria ", Service historique de la marine de Brest : 4 P 7236.

50. L'armement au bornage ne permet que des navigations d'un maximum de $80 \mathrm{~km}$ à partir du port d'attache. Il permet une sorte de cabotage de proximité effectué sur des bâtiments modestes. Cela permet également aux inscrits maritimes en attente d'autre chose d'accumuler les mois pour la caisse des invalides et donc leur retraite. 
par contre, très certainement l'ascendant de ses frères aînés qui, bien installés dans la carrière maritime, l'incitent sûrement à faire de même. Contrairement à ces derniers, c'est presque à l'âge " normal " qu'il embarque pour la première fois comme mousse vers Terre-Neuve en avril 1848. Il a quatorze ans. Ses deux frères ont embarqué avec lui sur le Saint-Brieuc pour une campagne à Terre-Neuve. Ils l'ont encouragé; on peut penser qu'ils le soutiennent et lui évitent trop de difficultés ou de brimades. Cependant, comme plusieurs de ses cousins ou neveux, il n'est pas convaincu par l'expérience. Il ne la renouvelle pas. Mais il lui faut bien gagner sa vie. Or, nous l'avons vu, à terre, c'est très difficile : la navigation de commerce pourrait peut-être lui convenir. Le plus simple, bien sûr, est de se tourner vers les armateurs du Légué qui ont souvent quelques petites unités réservées au cabotage. Alors, l'année suivante, en juin 1849, il embarque sur l'Industrie française, une goélette de l'armateur briochin Boullé. À part de brèves escales, il navigue presque sans discontinuer sur ce caboteur jusqu'en septembre 1852. Il découvre ainsi plusieurs ports de la Manche et de l'Atlantique de Rouen à Bayonne. En 1853, il embarque sur la goélette Gazelle du même armement puis sur le sloop Trois frères de l'armement Sebert. Il s'agit toujours d'aller chercher du sel dans les ports de l'Atlantique mais aussi de prendre du charbon au pays de Galles. En attendant son service militaire, c'est cette fois sur un caboteur malouin que Hyacinthe s'embarque. En août 1853, il débarque du Huron à Saint-Malo où il est levé pour trois ans par la marine d'état. À son retour en 1856, il est, lui aussi, un homme différent, qui a fréquenté d'autres ports et d'autres marins. Il a admiré les impressionnants vapeurs de la marine impériale. Il a entendu parler de ces grands voiliers qui sillonnent les mers du globe à destination de ports aux noms fabuleux. Dahouet doit lui sembler bien petit et ses horizons étriqués.

Il n'est pas le seul pour qui la marine au long cours est nimbée d'un grand prestige. Dans les milieux maritimes, on envie parfois ceux qui embarquent pour des pays lointains variés et attrayants, sur de grands navires qui ne sentent pas la morue. Les pêcheurs de Terre-Neuve ou d'Islande, à l'occasion de campagnes de cabotage ou lors de leur service dans la marine rencontrent d'autres marins, visitent d'autres ports et rêvent d'autres horizons s'imaginent un avenir meilleur. Certains, même s'il faut embarquer d'un port éloigné, franchissent le pas lorsque, par exemple, ils sont libérés des obligations militaires. Il faut dire que les circonstances les y incitent. Sur la côte bretonne alors que les ports continuent avec plus ou moins de succès leurs activités halieutiques traditionnelles, les autres secteurs sont en expansion. Le chemin de fer n'ayant pas encore pénétré la Bretagne, le petit cabotage continue à prospérer, permettant les échanges entre les villes côtières. Mais surtout, dopé par le développement de l'industrie, le commerce maritime international croît fortement : les armements au long cours et au grand cabotage se multiplient. Certains armateurs morutiers, comme nous l'avons noté savent en tenir compte en utilisant au mieux leurs navires l'automne et l'hiver. Quant aux grands ports, ils s'équipent 
de façon à recevoir des navires de plus en plus grands et de plus en plus nombreux ${ }^{51}$. À Nantes et à Saint-Nazaire plus tard, à Rouen et au Havre, les grands voiliers au long cours et les vapeurs ont besoin d'équipages plus nombreux : on recrute intensément.

En février 1857, Hyacinthe quitte Pléneuf pour Rouen où il s'embarque comme matelot sur le trois-mâts Franciscopolis. Il revient au Havre en décembre après un long périple qui l'a amené dans les ports du Pérou et du Chili. Convaincu par cette première expérience de la navigation au long cours, il fait six autres campagnes comme matelot sur des trois-mâts d'armements havrais. Les découvertes se succèdent. En 1858 et 1859, il fait escale à Valparaiso, à Sydney, à Papeete. En 1860, de nouveau à bord du Franciscopolis, il va à l'île Bourbon ${ }^{52}$. Fin 1860, à bord du trois-mâts Brune, il quitte Le Havre pour les Antilles, Cuba et le Vénézuéla : il est de retour au premier trimestre 1861. Le dernier voyage au long cours est interminable. D'août 1861 à octobre 1862, le trois-mâts Ville de Metz, à la recherche de chargements exotiques, fait le tour du globe en passant par Montevideo, l'île Maurice et Rangoun. Une apothéose, peut être, mais quinze mois, très loin du pays et de la famille, dans des conditions souvent difficiles avec des tensions qui s'accumulent ${ }^{53}$... Il faut une pause. Hyacinthe s'accorde alors six mois de relâche et décide finalement d'abandonner, à vingt-huit ans, une navigation au long cours dont, peut-être, il gardera malgré tout la nostalgie.

\section{Une ultime tentative}

Mais que faire? On peut croire qu'il a gardé un très médiocre souvenir de Terre-Neuve et qu'il n'est pas question pour lui d'y retourner. Par contre, au départ de Dahouët, il y a depuis peu une autre possibilité qui s'offre à lui : la pêche "à Islande ». Ses frères, ses amis lui en parlent. On lui fait valoir que d'aucuns y ont trouvé de bons revenus ${ }^{54}$. Les armateurs locaux sont toujours en quête de marins expérimentés. Hyacinthe se laisse convaincre, d'autant qu'on lui propose d'emblée le grade d'officier. En mars 1863, la goélette Saint-Guillaume de Jean-Pierre Le Péchon fait route vers l'Islande avec ses 19 hommes. Hyacinthe est lieutenant. L'automne et l'hiver suivants, il est de la campagne de cabotage sur la goélette Edmond de l'armement Boutevillain de Grandpré : livraison de morues à La Rochelle, chargement

51. À Saint-Nazaire, où Hyacinthe finira par s'installer, entre 1858 et 1867, tandis que les premiers paquebots apparaissent, le nombre de navires armés passe de 158 à 262 pour le long cours et de 36 à 66 pour le cabotage, Arch. dép. de Loire-Atlantique, Registres de désarmement, 7 R6 26 et 7 R6 27.

52. Il s'agit de La Réunion.

53. De nombreuses punitions, une bagarre au couteau... D'après le rôle de désarmement, Arch. dép. de Seine Maritime, 6 Mi 135.

54. Citons, par exemple, la fameuse formule des pêcheurs de Paimpol : " ar bara e oa du-hont ", " le pain était là-bas ", Jean-Loup AvRIL et Michel QuÉMÉRÉ, Pêcheur d'Islande, Morlaix, De mémoire d'homme, 1984, p 13. 
de sel à l'île de Ré. L'année suivante, sur le même navire, Hyacinthe va à nouveau à Islande et à La Rochelle. En 1865, il s'embarque sur la goélette Marguerite de l'armateur malouin Gauvelin. Ce sera sa dernière campagne à Islande. Manifestement, il n'a pas été plus convaincu que par la pêche à Terre-Neuve. Peut-être est-il un bon marin mais un piètre pêcheur. Or, les revenus de chacun, y compris des officiers, dépendent en grande partie du nombre de morues prises. Décidément la grande pêche ne lui convient pas. Fin 1866, Hyacinthe quitte la Marguerite qui est venu livrer sa morue à Bordeaux et rejoint Pléneuf. Là, il apprend que le trois-mâts Reine des anges, où son frère aîné est second, fera escale à Rouen après sa campagne à Terre-Neuve.

\section{Retour au commerce}

C'est donc sur ce navire de Jean-Pierre Le Péchon que Hyacinthe reprend sa carrière de marin au commerce de janvier 1866 à juin 1868 . Mais il ne veut probablement plus s'éloigner trop ou trop longtemps. Il va éviter les destinations au long cours et se consacrer au grand cabotage. En compagnie de son frère, il fait d'abord une longue campagne entre Manche et Méditerranée. Lorsque ce dernier débarque pour la dernière fois, Hyacinthe, qui est maintenant lieutenant, reste sur le même navire. Toujours avec le capitaine Ancelin, le navire est réarmé pour le cabotage en août 1867. Il va d'abord chercher du bois en Suède pour le livrer à Nantes puis est expédié sur lest à Swansea pour y chercher du charbon. Là-bas, Hyacinthe est peut-être surpris par la destination de la cargaison. En effet, sur le rôle d'équipage, une apostille ${ }^{55}$ signée par le consul de France, stipule que Hyacinthe " s'engage à suivre le navire au long cours moyennant une augmentation de salaire de 15 francs par mois ". Le charbon sera livré à Pointe à Pitre et le navire rentrera à Nantes durant l'été 1868 chargé de denrées coloniales.

Ce sera la dernière grande traversée pour Hyacinthe. À partir de ce moment, il se consacre au grand cabotage au service d'armements nantais et il revient fréquemment dans les ports de l'0uest. Dorénavant, il fréquentera le plus souvent les ports de Cardiff, Swansea, Bilbao et Nantes. À bord des bricks Visitation et Deux Marguerite, où Hyacinthe est maintenant second, on embarque des céréales pour la Grande Bretagne, du charbon pour Nantes ou pour Bilbao, des minerais pour l'Angleterre. Le commerce s'intensifie, les marchandises à livrer ne manquent pas. Aussi, les escales se font plus brèves et les rotations s'accélèrent. Or, Hyacinthe veut fonder une famille et il considère que ce mode de vie n'est pas compatible avec ce projet. En septembre 1872, il quitte définitivement le Bernigo à Nantes. Il a trente-huit ans. En octobre de la même année, il se marie à Saint-Nazaire avec Monique Durand. C'est dans cette ville en plein développement que le couple va désormais vivre.

55. Apostille sur le rôle de désarmement, Arch. dép. de Loire Atlantique 7 R 4561. 


\section{Atterrissage}

Hyacinthe a donc choisi de ne plus s'éloigner, de voir grandir les enfants qui vont naître mais, pour bénéficier de sa retraite, il a intérêt à rester inscrit maritime. Pendant dix ans, en compagnie d'un autre marin, il va donc pratiquer la pêche au poisson frais, s'éloignant peu de Saint-Nazaire où les canots Porcé puis Pilote d'intérieur sont réarmés chaque année. Il partage à part égale avec son compagnon, le fruit de leur pêche commune. Fréquemment au port, l'un et l'autre ne vivront plus les grandes traversées que par procuration.

En octobre 1883, ironie du sort ou signe des temps, alors qu'il a toujours navigué sur des voiliers, c'est sur un petit " vapeur " armé au bornage que Hyacinthe s'embarque une ultime fois à Saint-Nazaire. Il n'a pas encore tout à fait cinquante ans. Sur les rôles, il n'est que matelot mais peu importe puisqu'il ne s'agit plus que de terminer son année peut-être en compagnie d'amis marins. Fin décembre, il débarque du Beaver et entame sa retraite.

Durant ces années de petite pêche, Hyacinthe a probablement entretenu la mémoire de ses voyages passés. Tout compte fait, même s'il a dû y renoncer, il ne garde pas une mauvaise impression de la navigation au long cours. Le temps passe et les souvenirs se magnifient. En tout cas, il doit penser que pour un jeune garçon nécessiteux, c'est une solution correcte, au demeurant meilleure que la grande pêche. Dans le port de Saint-Nazaire où on peut voir des navires de plus en plus grands, de plus en plus nombreux, il a conservé des relations fort utiles. C'est ainsi que Hyacinthe fera venir quatre de ses neveux pour qu'ils embarquent, forts de ses recommandations, sur des vapeurs ou des voiliers de la marine de commerce. Mais ceci est une autre histoire.

\section{Un parcours familial}

\section{Deux beaux-frères différents}

Restant dans le cadre de Pléneuf, il est permis de s'interroger sur les carrières des beaux frères de Jean-Marie et de Joseph-Marie avec lesquels ces derniers sont allés vivre. Deux inscrits maritimes, acteurs de l'épopée de la grande pêche mais dont les carrières sont significativement très dissemblables. Deux exemples, deux stades d'évolution différents du passage de la terre à la mer.

Quand Jean-Marie Barbedienne se marie en 1844, il entre dans une famille voisine qu'il connaît fort bien. Son beau-frère, François Barbedienne*, de quelques années son aîné, a commencé sa carrière à Terre-Neuve, sur les morutiers du Légué, avant lui et a probablement joué un rôle dans son choix. Excepté pendant la période de son service militaire, François est, comme Jean-Marie, de toutes les campagnes de pêche à Terre-Neuve, sur des navires du Légué d'abord, ensuite sur des navires de Dahouët. Cependant, contrairement à son beau-frère avec qui il embarque parfois, 
sa carrière s'arrête tôt, en 1856, alors qu'il n'a que quarante-deux ans. C'est qu'il n'a pas fait le choix de la carrière maritime. Il n'en a probablement pas la nécessité, la pêche étant pour lui un appoint. Sa présence est nécessaire pour aider sa tante et sa sœur quelques mois par an, et pas une seule fois il n'embarque à l'automne, après la campagne de pêche. C'est un exemple de double activité durable. Il reste senneur, il ne prend pas de grade; de toute façon, il sait bien que dans ces conditions, il ne pourra jamais atteindre les annuités lui donnant accès à la demi-solde. Alors, il choisit son heure et quitte la pêche pour s'occuper de son lopin de terre au moment qui lui semble le plus opportun. C'est le cas en cette année 1864, alors que l'avenir de sa sœur et de son beau-frère semble définitivement assuré.

Joseph-Marie épouse Joséphine Audrain en 1860. Comme son frère aîné, c'est avec une voisine qu'il se marie. Comme lui également, il s'installe chez sa femme et cohabite avec un beau-frère marin et ami de longue date. En effet, Mathurin Lévêque avec qui il a déjà navigué plusieurs fois a épousé l'autre fille Audrain. Comme Joseph-Marie, Mathurin exerce d'abord diverses activités liées à la pêche sur la côte de Terre-Neuve. Plus rapidement que son beau-frère, il opte pour une véritable carrière maritime et, très vite, il est de toutes les campagnes, pêche à Terre-Neuve et cabotage. Comme son beau-frère aussi, il devient "islandais " et c'est également comme officier qu'il termine une carrière de marin à temps plein.

\section{Le passage au féminin}

Il n'est pas possible de clore cet article sans évoquer brièvement les sœurs Barbedienne au moins dans leurs rapports avec la mer. Bien sûr, à une époque où les femmes n'embarquaient jamais et où elles étaient confinées aux tâches ménagères, le problème de la transition se pose de manière très différente. C'est le mariage qui est alors déterminant même si, évidemment, il est influencé par les fréquentations de la famille; et celles-ci changent. Comme leurs frères, les sœurs Barbedienne ont eu une enfance de petites paysannes. Par la suite, elles ont rapidement été chargées de tâches domestiques ou agricoles mais ont connu, par l'intermédiaire de leurs frères, des marins de plus en plus nombreux.

L'aînée, Marie-Jeanne, après la mort d'un premier époux se remarie avec un autre laboureur Pierre-Marie Barbedienne* avec qui elle aura six enfants. Elle demeure donc attachée à la terre. Si, comme nous l'avons vu, le second de ses fils, Jean-François, fait l'essai d'un séjour à Terre-Neuve, il avait sûrement d'autres possibilités puisqu'il ne réitère pas l'expérience et retourne définitivement au village. Il rejoint ses frères et, à partir de ce moment, est recensé, ainsi que ces derniers, comme laboureur. De par son époux, Marie Jeanne est demeurée paysanne : le bref épisode maritime se referme rapidement et définitivement.

La seconde fille, Sainte, se marie avec un pauvre journalier venu de Plaintel. Ce sont eux qui maintenant prennent en charge les quelques bâti- 
ments et terres de la famille. Bien sûr, le couple retrouvera les difficultés de la génération précédente et vivra chichement de la terre. On verra alors se reproduire le même scénario qu'un quart de siècle auparavant sauf que, cette fois, les trois garçons iront chercher un embarquement directement sur des navires de commerce. Leur oncle Hyacinthe n'aura aucune difficulté à convaincre Sainte et Joseph d'envoyer leurs fils à Saint-Nazaire : il se considère comme bien placé pour choisir un bon début de carrière pour ses neveux. C'est dans ce port, comme leur cousin Joseph-Léon, que successivement, Joseph-Marie, Hyacinthe et Jean-Marie seront enrôlés comme mousses. Trois jeunes hommes qui auront peut-être des conditions de travail meilleures que leurs oncles mais dont les embarquements fréquents et lointains rendront les visites à Pléneuf rares.

Quant à Adélaïde, la plus jeune des filles Barbedienne, elle passe radicalement du côté de la mer. Une mer qui va détruire son existence. En effet, elle se marie avec un marin voisin, ami de ses frères, qui passe lui aussi de Terre-Neuve au commerce. Mais, contrairement aux frères Barbedienne, il n'ira pas au bout de sa carrière. Adélaïde apprend sa tragique disparition en mer, au large de la Martinique, moins de trois ans après la cérémonie. Son fils unique, Jean-Marie, est admis à l'école des mousses de la marine militaire à Brest. À dix-huit ans, il est timonier breveté et paraît bien parti pour faire carrière. Cependant, le sort s'acharne sur la pauvre Adélaïde : en 1882, son fils disparaît, à vingt-deux ans, au large de Trinidad.

En un demi-siècle, la famille Barbedienne est donc devenue presque intégralement une famille de marins. Était-ce inéluctable? De quel degré de liberté les acteurs de cette histoire ont-il joui dans leurs choix? Pour tenter de répondre, il faut analyser le déroulement des événements : les différents stades, les modalités et les causes des passages de l'un à l'autre, d'une part au niveau des individus, d'autre part au niveau de la famille. Enfin, il faudra envisager la valeur d'exemplarité de l'histoire de cette famille.

Et tout d'abord, est-il nécessaire, de passer par la grande pêche avant de devenir marin ${ }^{56}$ ? Bien sûr que non, mais dans la baie de Saint-Brieuc, c'est dans ce milieu qu'on recrute le plus, et ce sont généralement des enfants ou frères de marins comme Hyacinthe Barbedienne qui ont la possibilité d'être, d'emblée, orientés vers la marine de commerce. Donc, dans un premier temps, le jeune paysan qui devient mousse ou novice devient marin-paysan ou plutôt pêcheur-paysan à Terre-Neuve six mois sur douze. Les difficultés de l'agriculture en sont la cause. Partent là-bas des bouches à nourrir qui auraient pu tout aussi bien devenir pâtres ou domestiques dans d'autres fermes. Aucune compétence n'étant exigée au début, les départs se font à tout âge, quand les conditions à terre deviennent trop difficiles. Aucune carrière n'est alors envisagée, la situation, provoquée par les circonstances, est probablement vécue comme provisoire. D'ailleurs,

56. Le terme est pris ici dans le sens commun : un homme qui navigue beaucoup et dont les compétences sont nécessaires pour la manœuvre ou l'entretien du navire. 
l'expérience peut cesser après chaque campagne quand le jeune et ses parents considèrent que les inconvénients surpassent les avantages. C'est ce que nous avons vu avec les fils et neveux de Jean-Marie. Petit à petit, cependant, les tâches attribuées aux jeunes recrues deviennent plus gratifiantes, plus intéressantes, plus formatrices : les abandons se font plus rares. Ainsi, certains s'installent longtemps dans une situation de paysanpêcheur jusqu'à ce qu'ils considèrent que ce n'est plus de leur intérêt. C'est le choix de François Barbedienne* qui semble avoir à terre une autre source de revenus.

L'étape suivante, qui se fait généralement un peu après le service militaire, consiste à se lancer dans une carrière de marin-pêcheur. Le paysanpêcheur prolonge alors les campagnes de pêche par trois à six mois de navigation commerciale. Ce choix, qui est déterminé par la répartition des charges au sein d'une famille, doit se faire sans trop tarder. En effet, il faut avoir effectué vingt-cinq ans de pêche ou de navigation pour être demi-soldier $^{57}$ à cinquante ans. Pour les mêmes raisons, sauf rares exceptions, ce choix devient de plus en plus irréversible au fil des ans. On voit bien que le bref retour à la terre de Jean-Marie est provoqué par des circonstances exceptionnelles. Par contre, ce dernier choix étant fait, il est rare qu'il soit une étape avant la navigation au commerce : les habitudes sont prises, l'offre pour le cabotage pur est faible et les armateurs cherchent à garder les marins-pêcheurs expérimentés.

Les itinéraires personnels, on l'a vu, peuvent être fortement déviés par les aléas de la vie. Il convient donc de se poser les mêmes questions dans le cadre un peu plus large de la famille. Là, il est clair que la famille Barbedienne, totalement rurale au début, est amenée à chercher d'autres sources de revenus. Dans une étape intermédiaire, certains de ses membres restent pour s'occuper de l'exploitation tandis que d'autres, plus ou moins marins, apportent à la communauté quelques revenus. Mais là encore, ce stade de pluriactivité familiale n'est pas durable car, le plus souvent, les problèmes agricoles demeurent et les inscrits maritimes ont un choix à faire. Alors, les pêcheurs-paysans sont de plus en plus nombreux à opter pour la carrière maritime : seuls un ou deux membres de la famille restent sur place tandis que les autres devenus adultes se marient et quittent la ferme. La double activité, imposée par les circonstances, dure finalement peu. À la génération suivante, on est marin, commerçant, voire artisan.

Ainsi donc, tant pour les frères Barbedienne que pour la famille dans sa globalité, il n'y a pas eu de double activité durable. À Pléneuf, le statut de paysan-pêcheur serait-il rare? Pourtant, on a vu le cas du beau-frère François Barbedienne*. Et puis, on ne peut que se poser des questions sur les membres de ces équipages pléthoriques qui reviennent au pays en octobre et qui ne se réembarquent pas pour le cabotage. Travaillent-ils la terre? L'examen des carrières des inscrits maritimes devrait mettre en

57. Pour toucher la retraite. 
évidence l'existence de beaucoup de paysans-pêcheurs. Ce n'est pas le cas à Pléneuf au milieu du siècle. En effet, si nous examinons les carrières des inscrits résidant dans cette commune, nous constatons, sans surprise, que la grande majorité d'entre eux ont participé peu ou prou à la grande pêche : très rares sont ceux qui n'ont pratiqué que le cabotage ou la petite pêche. Considérant maintenant la catégorie la plus nombreuse, le registre ouvert en 1850, montre que moins de $10 \%$ d'entre eux ne font, comme François Barbedienne*, que les campagnes de pêche ${ }^{58}$. Tous les autres complètent plus ou moins par des embarquements au cabotage, à la petite pêche ou même au long cours. Il est, par ailleurs, exclu que l'existence de paysans pêcheurs non inscrits sur les registres de l'inscription maritime fausse les données précédentes. En effet, pour tout embarquement, l'inscription est exigée et on peut demeurer inscrit tout en ayant une activité marine très réduite. On est rayé officiellement après plusieurs années sans embarquement ou bien après déclaration officielle auprès du commissaire à l'inscription maritime. C'est ce que finissent par faire ceux qui ont fait ce choix de la double activité et qui, nous l'avons vu, cessent leur activité précocement et se déclarent alors laboureurs aux agents recenseurs : ils n'attendent pas l'âge d'une retraite dont ils ne pourront, de toute façon, pas bénéficier. En outre, au cours du siècle, ces paysans-pêcheurs qui n'ont jamais été très nombreux, deviennent de plus en plus rares. Le système de retraite ${ }^{59}$ pousse en effet de plus en plus clairement vers une décision précoce. De plus, la diminution des campagnes à Terre-Neuve au profit de l'Islande, qui est une école de formation marine intense et rapide, va dans ce sens.

Les conditions d'emploi étant les mêmes, on pourrait être tenté d'extrapoler les constatations précédentes à tous les ports morutiers de la baie de Saint-Brieuc et même de la côte nord de la Bretagne. Et que se passe-t-il là où il n'y a pas de grande pêche? Il est tentant d'établir un parallèle avec la côte sud de la Bretagne où la sardine ${ }^{60}$ a joué pour le développement économique de certaines régions côtières le même rôle que la morue sur la côte nord. Or, il a été montré que, à la même époque, là aussi, peu nombreux étaient les inscrits maritimes qui n'exerçaient que la pêche à la sardine en complétant leurs revenus par une activité terrienne. Tout au moins tant qu'elle n'était pas une source de revenus suffisants. La proportion est toutefois plus importante que sur la côte nord. C'est sans doute parce que la pêche à la sardine autorisait plus de souplesse que la pêche à la morue

58. Ultérieurement, c'est encore plus net : sur les 166 marins résidants de Pléneuf qui sont inscrits entre 1865 et 1869 , seulement 7 n'ont fait que des campagnes de pêche à la date d'ouverture du registre suivant en 1883.

59. C'est la caisse des invalides alimentée par l'État, les armateurs et les inscrits maritimes qui gère les pensions appelées demi-soldes. Il faudra attendre 1905 pour que les campagnes de pêche comptent pour années entières.

60. Dubors, Xavier-François, "L'évolution de la pratique de la pluriactivité chez les pêcheurs sardiniers en Bretagne au XIX ${ }^{\mathrm{e}}$ siècle : de la tradition à la nécessité ", dans : LE BouËDEC, Gérard, Ploux, François, CÉRIno, Christophe et Geistdoerfer, Aliette (dir.), Entre terre et mer. Sociétés littorales et pluriactivités ( $\mathrm{XV}^{\mathrm{e}}-\mathrm{XX}^{\mathrm{e}}$ siècle), Rennes, PUR, 2004, p. 217-226. 
qui, elle, imposait beaucoup de contraintes par sa durée et son éloignement. De toute façon, on constate que dans les deux cas, la tendance est à la mono activité quand c'est possible. La pluriactivité semble n'être qu'une parenthèse imposée par des circonstances défavorables.

Le dernier mouvement général auquel participent les Barbedienne, c'est le passage à la marine de commerce, la lente migration vers les grands ports qui en découle. Ce passage, qui suppose une certaine familiarité avec le milieu maritime, concerne des proches de marins confirmés. Il peut également concerner quelques matelots qui ont fortifié leur vocation pendant leur service à la marine. Cette fois, le changement est peut-être autant la conséquence de choix individuels que de la conjoncture économique. Il a lieu essentiellement durant la seconde partie du siècle au fur et à mesure que l'appel des grands ports se fait plus pressant et que les opinions se modifient. Ainsi, alors que pour Jean-Marie, le métier de marin ne se dissocie pas de la pêche au point qu'il tente d'entraîner fils et neveux dans la même voie, Joseph Marie, plus circonspect, est sensible à l'opinion de son jeune frère Hyacinthe qui a déjà franchi le pas. Il le charge de faire embarquer Joseph-Léon au long cours à Saint-Nazaire. Hyacinthe, devenu maintenant étranger au monde de la grande pêche va ainsi entraîner sur sa trace non seulement le fils de Joseph Marie mais aussi trois autres neveux, fils de sa sœur Sainte, qui n'auront pas la possibilité de vivre de la terre. Les trois, à treize ans, débuteront leur carrière au commerce à Saint-Nazaire. Quand, en 1872, un an après la mort de Jean-Marie, le premier de ses neveux s'embarque au long cours à Saint-Nazaire, l'intermède de la grande pêche est terminé pour les Barbedienne.

\section{Sources}

Service Historique de la Marine de Brest

pour les quartiers de Saint-Brieuc et de Brest

- Rôles de désarmement : 4 P 7129 à 4 P 7324

- Registres matricules : 4 P 3 : 79, 86, 119, 159, 160, 229

Service Historique de la Marine de Toulon pour le quartier de Marseille

- Rôles de désarmement : 13 P9 170 et 13 P9 342

Archives départementales de Seine-Maritime (Rouen)

pour les quartiers de Rouen et du Havre

- Rôles de désarmement : 6 P6 2376 Mi 120, 135 et 140

Archives départementales d'Ille-et-Vilaine (Rennes) pour le quartier de Saint-Malo

- Rôles de désarmement : 4 S 297 et 4 S 649

Archives départementales de Loire-Atlantique (Nantes)

pour les quartiers de Nantes et de Saint-Nazaire

- Rôles de désarmement pour Nantes : 7 R4 490,558, 561, 570 et 585 
- Rôles de désarmement pour Saint-Nazaire : 7 R 6 200, 206 et 216

- Registres de désarmements pour Saint-Nazaire : 7 R 6 26-27

État civil : archives départementales des Côtes-d'Armor (commune de Pléneuf) - État civil : 5 Mi EC : 81, 240,241, 1001 à 1004

- Recensement : 6 M 391 et 6 M 392

- Cadastre : 3 P 191/6 et 3 Q 2164

Archives municipales de Saint-Brieuc

- Correspondance du capitaine Conan : 10 Z 5

\section{Annexe - La famille Barbedienne de Pléneuf}

\begin{tabular}{|c|c|c|c|c|c|c|}
\hline \multicolumn{4}{|c|}{$\begin{array}{l}\text { Pierre Antoine Barbedienne } \\
\text { Né en } 1777\end{array}$} & \multicolumn{3}{|c|}{$\begin{array}{l}\text { Adélaïde Boschet } \\
\text { Née vers } 1785\end{array}$} \\
\hline \multicolumn{4}{|l|}{1} & \multicolumn{3}{|c|}{ I } \\
\hline \multicolumn{7}{|c|}{ | } \\
\hline I & | & | & | & | & | & I \\
\hline 1809 & 1817 & 1823 & 1825 & 1828 & 1829 & 1833 \\
\hline Pierre & Jean & Marie & Joseph & Sainte & Adélaïde & Hyacinthe \\
\hline Julien & Marie & Jeanne & Marie & & & \\
\hline+ & + & + & + & + & + & + \\
\hline Marie & Jeanne & Pierre & Joséphine & Joseph & Jean & Monique \\
\hline Sainte & & Marie & & Guillaume & Marie & \\
\hline Lévêque & Barbedienne* & Barbedienne* & Audrain & Auffray & Deguen & Durand \\
\hline I & I & | & I & | & | & \\
\hline I & I & 1 & i & i & i & \\
\hline 1840 & 1848 & 1851 & 1864 & 1859 & 1860 & \\
\hline Pierre & Jean & Jean & Joseph & Joseph & Jean & \\
\hline Marie & François & François & Léon & Marie & Marie & \\
\hline & & & & 1865 & & \\
\hline $\begin{array}{l}\text { es: seuls } \\
\text { Barbedien }\end{array}$ & $\begin{array}{l}\text { nt indiqués les } \\
\text { et de Adélaï }\end{array}$ & $\begin{array}{l}\text { s enfants de } P i \\
\text { de Boschet } q u\end{array}$ & $\begin{array}{l}\text { ierre } \\
\text { li ont }\end{array}$ & Hyacint & & \\
\hline $\begin{array}{l}\text { ge adulte. } \\
\text { qués ceux }\end{array}$ & $\begin{array}{l}\text { uant à leurs } p \\
\text { i apparaissen }\end{array}$ & $\begin{array}{l}\text { tits enfants, s } \\
\text { tans le texte }\end{array}$ & $\begin{array}{l}\text { seuls } \\
\text { Par }\end{array}$ & 1867 & & \\
\hline tous ceux & ui ont été mc & arins ou pêch & heurs & Jean & & \\
\hline aractères & as. Enfin, cor & nme dans le $t$ & texte & Marie & & \\
\hline
\end{tabular}




\section{RÉSUMÉ}

Cet article analyse à une échelle micro-historique le passage de la terre à la mer d'une famille de paysans bretons. Durant les premières décennies du $\mathrm{XIX}^{\mathrm{e}}$ siècle, cette famille s'enfonce progressivement dans la pauvreté. Cependant, sa situation géographique, en bordure de la baie de Saint-Brieuc, permet à trois garçons de trouver facilement une réponse provisoire et partielle à la précarité des revenus agricoles : l'embarquement saisonnier pour la grande pêche à Terre-Neuve. Assez rapidement toutefois, les circonstances et les nécessités les amènent à quitter définitivement la terre pour devenir pêcheurs puis marins. À partir de ce moment, formés par la grande pêche, ils participent aux différentes mutations de celle-ci : passage de Terre-Neuve à Islande, tentatives de diversification vers l'armement au commerce international. L'évolution ultime, dans la deuxième partie du siècle, amènera le plus jeune frère à participer à la lente migration des inscrits maritimes de Bretagne vers les grands ports de commerce alors en forte expansion. Les parcours des trois frères s'inscrivent donc dans l'évolution générale des pêcheries et du commerce breton au XIX ${ }^{\mathrm{e}}$ siècle.

\section{ABSTRACT}

This article analyzes, on a microhistoric scale, the passage of a Breton peasant family from the land to the sea. In the first decades of the $19^{\text {th }}$ Century, this family progressively slipped deeper into poverty although their geographic location, right on the bay of Saint-Brieuc, made it possible for three boys to easily find a temporary, partial answer to the insecurity of agricultural revenue: seasonal departures to fish on Newfoundland's coast. However, circumstance and need quickly led them to leave the land permanently in order to become first fishermen, then sailors. From this moment onward, trained in sea fishing, they participated in its different transformations: passage from Newfoundland to Iceland, and attempts at diversification towards international trade. The last evolution, in the second part of the century, led the youngest brother to participate in the slow migration of the naval enlisted men of Brittany towards commercial ports, at that time rapidly expanding. The experiences of the three brothers thus fit into the general evolution of fisheries and Breton commerce in the $19^{\text {th }}$ Century. 